\title{
Le cyberprix
}

\author{
William Menvielle, Denis Pettigrew et Jocelyn D. Perreault ${ }^{1}$ \\ Université du Québec à Trois-Rivières
}

Les chantres du commerce électronique ont longtemps laissé croire, aux entrepreneurs d'une part et aux particuliers de l'autre, que l'Internet allait apporter de bien meilleurs prix aux produits vendus par ce mode de distribution en raison d'une éviction des intermédiaires traditionnels. Hélas, la réalité à laquelle nous sommes confrontés est quelque peu différente de cette théorie, même si quelques modèles d'affaires proposent un privilège à l'acheteur.

Comme vous l'avez compris, dans ce deuxième article ayant pour thème l'intégration du marketing-mix dans Internet, nous étudierons la variable prix. À l'instar du monde physique, on retrouve dans Internet les mêmes caractéristiques de cette variable: il est possible d'obtenir des conseils, des produits et des services gratuits ou payants. Toutefois, la particularité d'Internet est d'avoir généré ses propres modèles d'affaires électroniques.

À cet égard, nous considérerons Internet à la fois comme un moyen de communication - et c'est sans doute l'aspect le plus souvent évoqué -, comme un moyen de distribution pour télécharger des logiciels ou de la musique, mais également comme un moyen de paiement, lors de l'achat en ligne notamment. Ces trois fonctions sont les plus communément recensées par de nombreux auteurs.

Ainsi, nous nous proposons, à partir des travaux de Timers ${ }^{2}$, de présenter les principaux modèles existants dans Internet. Par la suite, nous définirons en quoi consistent les notions de gratuité et de payant avant de voir les relations entre la variable prix et les modèles d'affaires. Notons finalement que notre approche se concentre aussi bien dans une perspective d'entreprise à entreprise $\left(\mathrm{B} 2 \mathrm{~B}^{3}\right)$ que de relation entreprise à consommateur $\left(\mathrm{B} 2 \mathrm{C}^{4}\right)$.
Sans pour autant proposer de nouveaux modèles de gestion ni de nouveaux paradigmes du marketing, mais simplement, à travers une revue de littérature et une série d'exemples, nous mettrons en lumière les enjeux de cette nouvelle tendance. Nous nous proposons dès à présent de nous intéresser au prix, après avoir abordé les nouveaux modèles d'affaires de l'Internet.

\section{Q uelques modèles d'affaires électroniques}

Dans la présente partie, nous proposons de présenter succinctement les différents modèles de commerce électronique existant à l'heure actuelle. La classification nous apparaissant la plus adéquate est celle de Timers ${ }^{5}$, qui présente les modèles d'affaires selon deux axes: leur degré d'innovation, en abscisse, et leur niveau d'intégration fonctionnelle, en ordonnée (figure 1).

Avant d'entrer plus amplement dans les détails, il conviendrait de donner les définitions des concepts auxquels nous recourrons dans le présent article. Notons au préalable que ces modèles se catégorisent suivant leur niveau de contenu, de transaction et d'interactivité. De façon globale, nous passerons en revue les boutiques électroniques et les centres commerciaux virtuels, les enchères électroniques, l'approvisionnement électronique et le courtage d'information.

Les boutiques électroniques (boutiques-é) représentent une nouvelle façon, pour une société ou un magasin, de faire du marketing sur le Web. En premier lieu, l'intégration d'un site Web est une façon de promouvoir l'activité de l'entité économique, bien au-delà des frontières de l'organisation et de son rayon d'activité. À un premier stade, on voit apparaître l'information électronique, puis progressivement, le site peut se transformer en site interac- 


\section{Figure 1. Les modèles d'affaires électroniques}

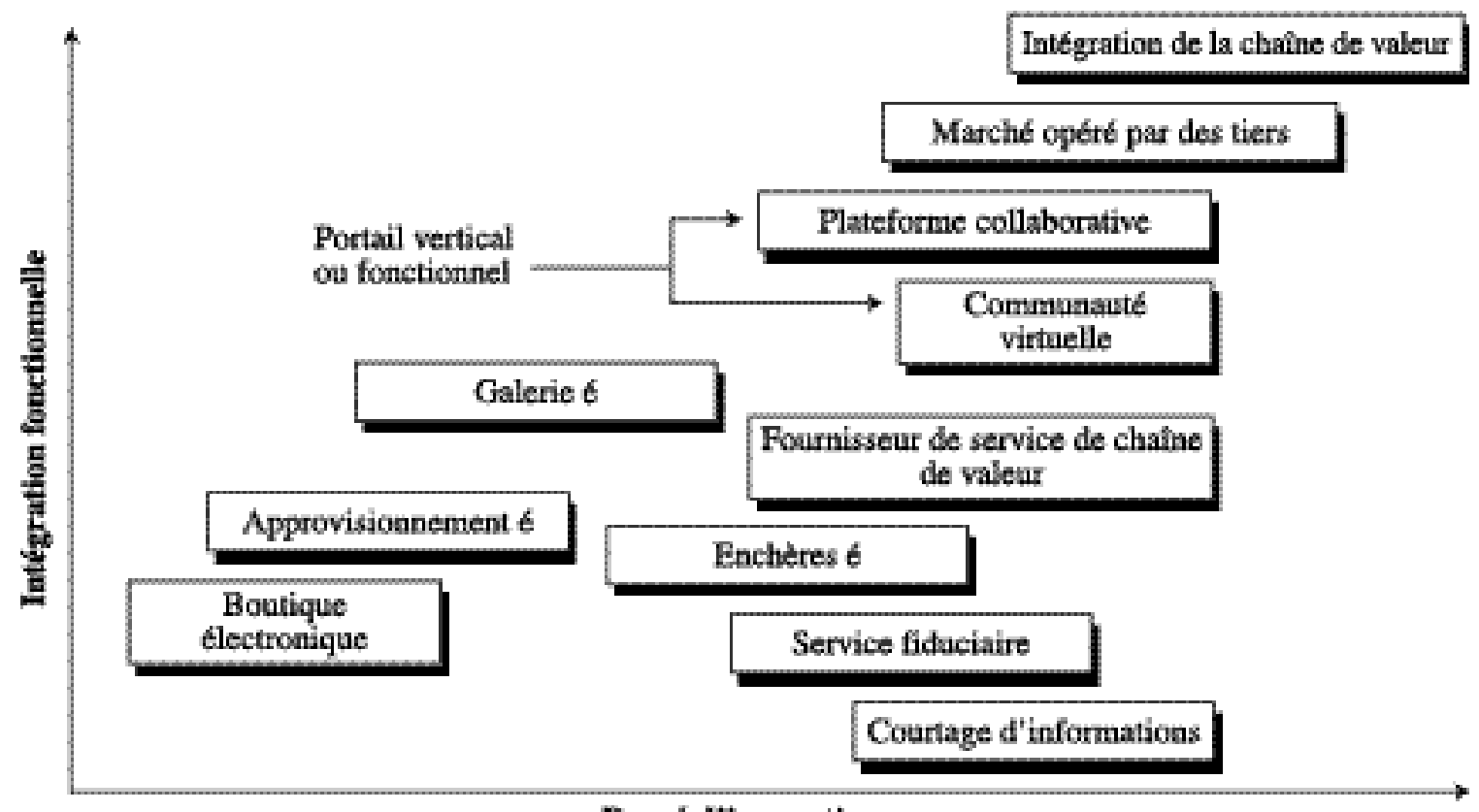

Degré d'innovation

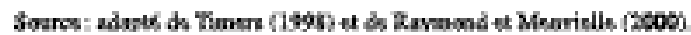

tif voire transactionnel. Pour l'entreprise, on souligne alors une réduction des coûts marketing, notamment pour ceux liés à la promotion et aux ventes. De son côté, le consommateur bénéficie d'un accès illimité en temps (magasinage virtuel), et d'une offre parfois plus diversifiée par exemple. On assiste progressivement à la naissance d'un marketing «one-to-one $»^{6}$, bénéfique à la fois au consommateur et à l'entreprise.

L'approvisionnement électronique regroupe les appels d'offres et l'approvisionnement électronique. Ce modèle peut inclure de la négociation, de la contractualisation et de la collaboration des différents acteurs. Le cas du site Merx $^{7}$ du gouvernement du Canada est éloquent à cet égard. Les avantages recherchés sont de pouvoir disposer d'un plus grand nombre de fournisseurs potentiels, et cette concurrence entraîne une baisse des coûts. De plus, au niveau des services, et de façon à s'approprier le marché, les entreprises, en lice pour décrocher un contrat, s'engagent à proposer un service de meilleure qualité ainsi que des délais de livraison améliorés. À cet effet, les négociations électroniques, de même que le travail en collaboration de la part de plusieurs acteurs, permettent de diminuer les délais de réalisation du contrat tout en améliorant encore le coût global.
De leur côté, les enchères électroniques (encan-é) correspondent à l'implantation électronique du mécanisme d'enchères. L'avantage de cette virtualisation est qu'elle permet d'inclure une présentation multimédia des biens offerts. Chez les consommateurs, les sites canadiens les plus célèbres sont Encan ${ }^{8}$ ou encore 123vendu.com ${ }^{9}$, alors que le même système existe pour les entreprises, où dans le domaine des pièces électroniques, Fastparts ${ }^{10}$ est un exemple de réussite typique. Le principe des enchères électroniques s'apparente à celui de la réalité, avec une mise, la rédaction d'un contrat, un paiement et la livraison du bien "gagné ». Bien évidemment, pour l'acheteur comme pour le vendeur, les avantages sont avant tout d'ordre matériel: économie d'argent, économie de transport (plus besoin d'apporter la marchandise chez un encanteur tant qu'elle n'est pas vendue) et économie de temps.

À un niveau d'intégration fonctionnelle supérieure, on trouve le centre commercial électronique (mall-é ou galerie-é) qui, à l'instar d'un centre commercial fait de «brique et de béton ${ }^{11} »$, regroupe un ensemble de boutiques (électroniques cette fois), ordinairement mises en valeur par un thème commun, par exemple une marque très connue, pouvant inclure une méthode commune - garantie - de paiement. Les avantages pour les différents acteurs de ce 
modèle d'affaires s'apparentent à ceux décrits précédemment pour les boutiques électroniques. À titre d'exemple, citons le site canadien Mall Canada ${ }^{12}$ et le centre d'achats électronique Fortune $1000^{13}$.

Enfin, le courtage d'information se compose d'un ensemble de services informationnels qui ajoutent de la valeur aux données, par exemple la veille stratégique, les profils de clientèle, le courtage d'opportunités d'affaires et le conseil en investissement. Diverses sociétés se sont spécialisées dans cette activité, mais il est certain que les cabinets d'audit et de conseils ont bénéficié de l'ouverture d'Internet. Citons à cet égard le Knowledgespace $\mathrm{d}^{\prime}$ Arthur Andersen. Timers ${ }^{14} \mathrm{y}$ intègre également les moteurs de recherche traditionnels tels qu' Altavista ${ }^{15}$ et Copernic ${ }^{16}$. Par extension, des moteurs de recherche spécialisés, véritables agents intelligents de veille ${ }^{17}$, se sont développés dans des fonctions comme la recherche de produits spécifiques ou simplement la recherche du meilleur prix offert pour un produit ou un service.

Les modèles d'affaires électroniques représentent un ensemble assez hétérogène, dans lesquels les entreprises peuvent agir parfois à divers niveaux à la fois (boutique électronique et communauté virtuelle).

Comme nous venons de les passer en revue, les modèles d'affaires électroniques représentent un ensemble assez hétérogène, dans lesquels les entreprises peuvent agir parfois à divers niveaux à la fois (boutique électronique et communauté virtuelle). Notre but était de montrer la diversité existant parmi tous ces modèles. Disons simplement que ceci posé, il est intéressant de s'attarder sur les véritables enjeux que pose le commerce électronique à l'entreprise et de voir comment une variable du marketingmix comme le prix s'intègre dans cet ensemble.

\section{La variable «prix»}

La viabilité d'une activité commerciale dans Internet dépend de son prix, mais il est de moins en moins considéré comme une condition nécessaire et non suffisante pour rester en course ${ }^{18}$. Différents systèmes se rencontrent dans Internet, depuis la gratuité jusqu'au paiement. Dans la présente partie, nous nous intéresserons uniquement au prix du produit acheté en ligne. Nous ne traiterons pas de la question relative à la fiscalité, ni des moyens de paiement et de leur sécurité.
Le choix d'un modèle d'affaires apparaît ici inévitable selon le type d'activité économique que l'on désire entamer sur Internet. Ainsi, selon que l'on diffuse gratuitement des données ou que l'on propose des produits payants ou de l'information moyennant rémunération, le type de modèle d'affaires est différent.

\section{La gratuité}

Ce sont principalement les services et l'information qui sont offerts gratuitement dans Internet. À cet égard, deux types de sites sont à distinguer. Dans un premier temps, les sites publics ou parapublics émanant d'un niveau de gouvernement, d'une institution de recherche (université) ou encore d'une quelconque organisation non gouvernementale entrent dans cette catégorie. Ces sites proposent principalement de l'information à télécharger sous la forme de brochures ou de cahiers.

Concernant les universités, leurs centres de recherche mettent souvent à disposition des résultats de recherche qu'ils offrent sous la forme de cahiers tout à fait gratuitement. Par exemple, l'Institut de recherche sur les PME propose la plupart de ses publications en ligne ${ }^{19}$. Les gouvernements proposent plutôt de l'information générale pour les consommateurs et les entreprises, mais là encore la gratuité permet au plus grand nombre, sans aucune restriction, de bénéficier de l'accès au produit. La gratuité de cette information obéit ainsi à la mission première de ces institutions: être au service de la communauté. Le site de Communication Québec ${ }^{20}$ dispense des brochures gratuites permettant aux citoyens d'obtenir de l'information sur les formalités à respecter dans le cas de la naissance d'un enfant par exemple.

Dans un second temps, les entreprises proposent, elles aussi, en partie toutefois, des produits gratuits. Leur stratégie est toutefois différente dans la mesure où elles ne dévoilent qu'une partie du produit pour que le consommateur $^{21}$ fasse l'acquisition du reste. Ainsi, la firme de sondage Internet $\mathrm{Nua}^{22}$ diffuse gracieusement des résumés de ses études dont les rapports sont disponibles dans leur intégralité sur le site, moyennant quelques milliers de dollars. Cette stratégie est notamment suivie par plusieurs firmes de consultants. Elle vise d'ailleurs à attirer le plus grand nombre de clients et à les inciter, tôt ou tard, à s'abonner pour avoir accès à l'intégralité de l'information.

Ainsi, pour proposer une offre gratuite, seuls quelques modèles comme les communautés virtuelles ou les portails 
d'information généraux ${ }^{23}$ ou spécifiques ${ }^{24}$ principalement $^{2}$ financés par de la publicité fonctionnent selon ce principe. En parallèle, bien évidemment, les modèles payants existent sous de nombreuses formes.

\section{Le payant}

Bien évidemment, il n'y a rien de révolutionnaire à affirmer qu'à l'instar du monde physique réel, le monde virtuel propose des produits et des services payants. L'achat d'un livre ou d'un disque compact chez Archambault $^{25}$, d'un chien ${ }^{26}$, d'outillages ${ }^{27}$ ou même d'une voiture (prochainement disponible sur le site de Ford Canada pour la province de l'Ontario) nécessite, en contrepartie, non plus une monnaie «sonnante et trébuchante » mais électronique, puisque cet adjectif accompagne désormais tout le monde de l'Internet. On se trouve donc ici dans le paradigme des célèbres boutiques électroniques, voire des rares centres commerciaux virtuels, mais pour ceux uniquement qui ont franchi les deux premières étapes des sites Web : aspect informationnel et interactionnel pour se rendre à l'étape ultime de l'aspect transactionnel.

Dans le cadre de la diffusion d'information toutefois, le cyberconsommateur n'a pas encore intégré le fait qu'il doive payer pour avoir accès à cette information. D'ailleurs, comme le rappelle Schwartz ${ }^{28}$ : «That's the first principle of Webonomics : Consumers will rarely pay a subscription fee for access to a Web site ». Une explication peut être donnée à ce phénomène par le fait que l'information divulguée dans Internet est majoritairement gratuite, que de nombreux fournisseurs d'accès gratuits ont fait leur apparition, si bien que le consommateur dresse un parallèle et rechigne à débourser pour des nouvelles, l'accès à des sites de clavardage ${ }^{29}$, le téléchargement de logiciels ou de musique.

Petit à petit, toutefois, les consommateurs acceptent le fait que certains sites soient d'accès payant. Et la révolution apparaît lorsque les modèles d'affaires (voir article Le cyberproduit, p. 55) proposent plusieurs modes de paiement. Ainsi, on a vu apparaître trois types de paiement: le paiement global, le paiement indirect et le paiement ponctuel ${ }^{30}$.

Le paiement global consiste à payer un abonnement pour avoir accès à un contenu informationnel, pouvant dans certains cas être diffusé sur un autre support. C'est le cas de l'abonnement à certaines bases de données, dont le support est parfois physique (CD-ROM, par exemple). De nombreux journaux procèdent de la sorte. On se trouve alors dans un modèle d'affaires de courtage d'information, dans lequel le consommateur paie en échange d'un service. Les industries de l'immatériel pourraient, dans un avenir proche, être intéressées par cette formule en proposant des abonnements à leurs services, moyennant un volume d'heures de consultation des documents, des téléchargements offerts à chaque mise à jour. On peut alors imaginer qu'un forfait Microsoft existe, par lequel entreprises et consommateurs pourraient obtenir les dernières versions des logiciels, l'accès à des dépannages en ligne et la consultation des ouvrages de référence. C'est donc un nouveau paradigme qui apparait, dans lequel toutefois ces entreprises ne font qu'une entrée timide. Microsoft $\mathrm{f}^{31}$ ne propose que la mise à jour de certains fichiers ou utilitaires; il n'est pas encore question de pouvoir obtenir en ligne la dernière version de «Windows». Sans doute, l'amélioration de la bande passante de l'Internet et l'ouverture d'esprit des internautes permettront cette audace.

De son côté, le paiement indirect consiste à établir une distinction entre le produit et le service que l'on met en ligne d'une part, et l'information que l'on diffuse sur ce produit ou ce service d'autre part. Dufour précise à cet égard que dans le cadre d'une librairie virtuelle, il est facile d'imaginer qu'un Internaute doive payer, ne seraitce qu'un coût minime, pour consulter la base de données de cette librairie sur la disponibilité d'un ouvrage, obtenir de l'information sur son prix, pouvoir lire le quatrième de couverture et d'un autre côté, réaliser une transaction intégrale dans le cas où cet ouvrage l'intéresserait et qu'il voudrait se l'approprier. Chez Amazon, on ne distingue pas ces deux services, et l'achat d'un ouvrage couvre les frais de promotion en quelque sorte.

Finalement, le paiement ponctuel est relatif à la consultation (pay per view) ou à l'usage (pay per use) (2) $^{32}$. Ce type de paiement est utilisé pour de petites sommes d'argent, et lorsque le consommateur désire une information bien spécifique dans le cadre d'un tout (ex.: achat d'un article précis dans une revue, ce qui n'est pas possible avec son homologue physique). Ainsi, dans le domaine académique, la consultation d'un papier de la célèbre Harvard Business Review se facture par article désiré. De la même façon, l'accès aux archives de la revue $L^{\prime}$ Actualité ${ }^{33}$ se fait moyennant la consultation d'une base de données exigeant l'inscription de son numéro de carte de crédit. Bref, le consommateur paie uniquement pour ce qu'il cherche, ce dont il a besoin. $\mathrm{Ce}$ 
principe peut donc paraître plus équitable; la publicité des revues est toutefois nécessaire pour financer un certain nombre de coûts. Seule la dématérialisation totale de ces produits permettrait de facturer des articles à l'usage, mais il faudrait imaginer alors la disparition du papier, à moins que les éditeurs optent pour l'intégration de la publicité sur Internet, ce qui est en voie de développement.

\section{La relation entre prix et modèle d'affaires}

De la gratuité d'un côté au produit payant de l'autre s'échelonne le long d'un continuum tout un ensemble de modèles de gestion du prix (gratuité, prix proposé par le consommateur, prix négocié, prix fixé par le manufacturier). En effet, comme le précise Reynolds ${ }^{34}$, des modèles d'affaires comme les sites d'enchères ou encore les catalogues ne peuvent gérer le prix de la même façon. Deux aspects sont à considérer : celui où le commerçant fixe les prix, qu'il modifie en fonction du client, et celui où le consommateur propose les siens.

\section{De la gratuité d'un côté au produit payant \\ de l'autre s'échelonne le long d'un continuum tout un ensemble de modèles de gestion du prix (gratuité, prix proposé par \\ le consommateur, prix négocié, prix fixé par le manufacturier).}

\section{Les prix émanant des commerçants}

La personnalisation de l'offre et l'analyse du comportement du consommateur ont permis au vendeur de discriminer l'offre par les prix ${ }^{35}$. Les modèles de gestion dynamique permettent de proposer un prix changeant dans le temps et cette fluctuation dépend du nombre de ventes effectuées: si le produit rencontre des acheteurs, le prix augmente, si le produit ne trouve pas preneur, le prix baisse jusqu'à ce que quelqu'un le trouve suffisamment bas pour acheter. Cette pratique existe bien plus souvent qu'on ne pourrait se l'imaginer, y compris pour des produits peu chers. À titre indicatif, certains livres sont offerts selon ce principe sur le site Site Sell ${ }^{36}$.

Concernant les catalogues ou les traditionnels sites de commerces permettant l'achat en ligne, à l'inverse, tous les clients ont droit au même prix, et les rabais et les promotions sont proposés à tous en même temps. Ainsi, deux stratégies sont possibles pour un modèle de boutique électronique.

\section{Les prix proposés par les consommateurs}

À l'inverse, dans le cadre des enchères électroniques, c'est le consommateur qui fixe le prix auquel il est prêt à acheter le produit proposé à la vente. En fonction des mises, il y a ou non surenchère sur le produit en question. Peu de différences notoires sont à souligner par rapport à son équivalent réel; l'avantage des enchères électroniques est d'éviter des coûts de transport tant que la marchandise n'a pas trouvé preneur. Par ailleurs, en raison de la publicité qui subventionne ce type de modèle d'affaires, il permet de proposer à la vente des produits parfois uniques ou en très petite quantité, comme des surplus d'inventaire.

En parallèle de ce modèle, l'approvisionnement électronique (ou les appels d'offres électroniques) n'est autre que la version virtuelle des célèbres appels d'offres. En lieu et place d'un produit, il s'agit d'un service qui est mis aux enchères et le vainqueur, cette fois, est celui qui aura proposé le prix le plus bas pour réaliser le contrat décrit dans l'offre. Il convient de distinguer deux types d'appels d'offres. Il y a les appels d'offres privés disponibles pour les fournisseurs de façon ciblée ainsi que les appels d'offres publics disponibles pour tous les fournisseurs ayant accès à la plateforme d'affaires (exemple de Merx précédemment cité). Ce sont le plus souvent les grandes entreprises ou les administrations publiques qui ont recours à ce type de plateforme. Toutefois, les PME peuvent aussi prendre part à ces marchés. Les bénéfices que les demandeurs en retirent résultent de leur capacité à obtenir une multitude de soumissions de la part de PME sous-traitantes; ceux qui ont lancé l'appel d'offres n'ont alors qu'à faire leur choix en considérant l'aspect compétitif du coût et certains critères de qualité. Les bénéfices sont importants pour les PME car elles sont en mesure de profiter de nouvelles opportunités d'affaires, de diminuer les coûts de soumission et de collaborer avec d'autres sous-traitants. Pour les appels d'offres privés, l'exemple de Trading Process Network Register ${ }^{37}$, une filiale commerciale de General Electric, est éloquent.

Par ailleurs, un autre modèle d'affaires est le RFP ${ }^{38}$. Le consommateur est l'acteur prédominant $\left(\mathrm{C}^{2} \mathrm{~B}^{39}\right)$ et recherche des offres commerciales (flux informationnel) via un site approprié, fixe son offre en termes de caractéristiques (prix maximum) et reçoit en échange les propositions des entreprises. En fonction des réponses, il choisira les produits ou services correspondant le mieux à ses attentes et réalisera la transaction (flux de marchan- 
dise de la part de l'entreprise et monétaire émanant du consommateur). Parmi les exemples de telles relations, on note des sites comme Priceline ${ }^{40}$ ou FreeMarkets ${ }^{41}$. Sur ce type de modèle, les transactions sont longues (plusieurs semaines ou plusieurs mois) et parfois soumises à des contraintes figées de la part de l'entreprise, notamment en matière de volume à acheter.

Dans le secteur de l'hôtellerie, certaines entreprises ont opté pour ce système, certainement pour répondre à la baisse de demande lors de la saison morte. Ainsi, le site permet au client de faire une offre dite raisonnable pour une chambre, en retour de quoi l'entreprise offre ou s'abstient de louer au demandeur. C'est le principe que propose l'auberge L'Estérel ${ }^{42}$, membre de la chaîne Hôtellerie Champêtre, qui stipule explicitement sur sa page de proposition de prix le message suivant: «Faites-nous une offre. Dites-nous le prix que vous voulez payer par jour, par chambre. Repas et activités non inclus».

Finalement, un autre modèle offre la possibilité au consommateur de transiger via des agents intelligents, que ce soit pour comparer des prix ou faire des enchères ${ }^{43}$. Raymond et Menvielle ${ }^{44}$ recensent deux types d'agents à ce niveau : les agents de comparaison des prix et les agents de négociation.

Les agents de comparaison de prix ${ }^{45}$ permettent d'identifier les prix des produits recherchés et d'identifier les fournisseurs qui les vendent et qui proposent le prix le plus compétitif. Les logiciels les plus connus sont BargainFinder, de la firme de consultants Andersen, ou encore $\mathrm{Jango}^{46}$. Bien avant, la célèbre université du Michigan (MIT) avait développé les premiers modèles d'agents, et même s'ils font aujourd'hui figure d'ancêtres, la déontologie veut que nous les citions. Il s'agit de Kasbah $^{47}$ et de Tête-à-Tête ${ }^{48}$. Ces agents intelligents s'avèrent utiles tant pour les particuliers que pour les entreprises, leur faisant économiser temps et argent à la recherche de «l'offre du siècle». À l'heure actuelle, la préférence va à des logiciels d'aspect plus convivial comme Copernic Shopper ${ }^{49}$.

Les agents de négociation sont efficaces sur les sites d'enchères électroniques. Les agents participent pleinement au processus de négociation. Auction Bot $^{50}$ est un agent permettant de réaliser des enchères électroniques. L'avantage de cet outil est que l'on peut entièrement

Tableau 1. La relation entre le type de prix et les modeles d'affaires de l'entreprise

\begin{tabular}{|c|c|c|c|c|}
\hline $\begin{array}{l}\text { Modèles } \\
\text { d'altaires }\end{array}$ & $\begin{array}{l}\text { Unité } \\
\text { temporelle }\end{array}$ & Type de prix & $\begin{array}{l}\text { Produits 6́changés } \\
\text { sur ce modêle d'affaires }\end{array}$ & $\begin{array}{l}\text { Fxemples camadiens ou } \\
\text { québécois d'applications }\end{array}$ \\
\hline Prix dynamique & $\begin{array}{l}\text { Temps réel } \\
\text { Nomibreax } \\
\text { echanges }\end{array}$ & $\begin{array}{l}\text { Volatile, } \\
\text { en temps reel }\end{array}$ & $\begin{array}{l}\text { Catćgorie restreinte } \\
\text { de produits, comme } \\
\text { les livres }\end{array}$ & http:J/www sitesell.com \\
\hline $\begin{array}{l}\text { Catalogue } \\
\text { électronique, centre } \\
\text { commercial virtuel }\end{array}$ & $\begin{array}{l}\text { Commandes } \\
\text { récurrentes }\end{array}$ & $\begin{array}{l}\text { Standard ou } \\
\text { négocié ats départ }\end{array}$ & $\begin{array}{l}\text { Produtits standards, } \\
\text { large choix }\end{array}$ & $\begin{array}{l}\text { http://www.canadiantire.ce } \\
\text { https/fwww.malleanada.com }\end{array}$ \\
\hline $\begin{array}{l}\text { Enchères } \\
\text { electroniqques }\end{array}$ & $\begin{array}{l}\text { Echanges peu } \\
\text { frequents }\end{array}$ & $\begin{array}{l}\text { Grande disparité } \\
\text { dependamment du } \\
\text { produit et des offreturs }\end{array}$ & $\begin{array}{l}\text { Produtits standards } \\
\text { ef non-standards, } \\
\text { equipernents usagés }\end{array}$ & htlp:l/encan canoe.com \\
\hline $\begin{array}{l}\text { Approvisionatement } \\
\text { electronique }\end{array}$ & $\begin{array}{l}\text { fehanges peu } \\
\text { frequents }\end{array}$ & $\begin{array}{l}\text { Grande disparité } \\
\text { dependamment du } \\
\text { produit et des offreurs }\end{array}$ & $\begin{array}{l}\text { Service ou prodait } \\
\text { complexe, sur mesure }\end{array}$ & http//www.merx.ca \\
\hline $\begin{array}{l}\text { Demande de } \\
\text { proposition de prix }\end{array}$ & $\begin{array}{l}\text { Transactions } \\
\text { de plusieurs } \\
\text { semaines ou mois }\end{array}$ & Prix sur mesure & $\begin{array}{l}\text { Produtits ef services } \\
\text { complexes, spécifications } \\
\text { en fonction des clients }\end{array}$ & http://www esterel com \\
\hline Agents intelligents & Temps réel & $\begin{array}{l}\text { Fixé au départ } \\
\text { par l'tutilisateur }\end{array}$ & $\begin{array}{l}\text { Produtits ef services } \\
\text { complexes, specifications } \\
\text { en fonction des clients, } \\
\text { equipernent usage }\end{array}$ & $\begin{array}{l}\text { http://www.copernic.com/ltr/ } \\
\text { products/shopperfindex htmil }\end{array}$ \\
\hline
\end{tabular}

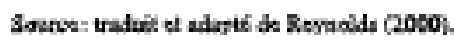


paramétrer le type d'enchères que l'on désire (temps, type d'offres...). Â la différence des autres logiciels de ce type, Auction Bot possède une interface personnalisable qui lui permet, une fois renseignée, d'agir de façon autonome. Il agit ainsi en tant que mandataire pour le client qui l'utilise.

Le paradoxe de ces modèles d'affaires est qu'ils ont justement été abandonnés en raison «du coût du temps passé dans la négociation $»^{51}$. Le marchandage des prix, laissé à la discrétion des pays du tiers-monde (notamment dans les souks), revient en force dans cette économie de l'information et du savoir. Mais peut-être n'est-ce qu'un phénomène cyclique, pérenne?

Quoi qu'il en soit, ces nouvelles formes de modèles d'affaires, dans lesquels le client est passé du stade passif à celui de proactif, changent la donne et renvoient le monde physique à la préhistoire des relations acheteursvendeurs. Désormais, le client dispose d'un pouvoir d'autant plus important qu'il joue sur la seule variable considérée par l'entreprise comme un revenu et non comme un coût ${ }^{52}$ : le prix. En réponse à cette tendance, l'entreprise, en proposant la vente via les nouvelles technologies, permet de réaliser des gains en baissant ses coûts de production, de distribution et de promotion, bref certains coûts de transaction rééquilibrant ainsi la relation. Nous avons synthétisé nos propos dans le tableau 1 ci-après, qui résume les éléments que nous venons de mentionner.

\section{Conclusion}

$\mathrm{Au}$ terme de cette brève argumentation, plusieurs questions se posent et nombre de lecteurs se demandent certainement, à l'instar de Smith, Bailey et Brynjolfsson ${ }^{53}$, ce qu'il en est des prix, de leur élasticité, de la stratégie pour compenser les coûts et de la dispersion des prix.

Ainsi quatre grandes questions se posent :

- Les prix sont-ils généralement moins élevés sur Internet?

- Les cyberconsommateurs sont-ils plus sensibles à de timides modifications du prix sur Internet?

- Les commerçants ajustent-ils leur prix plus souvent et plus sensiblement sur Internet?

- Les écarts entre les prix les moins élevés et les plus élevés sont-ils moins importants sur Internet?
- Les prix sont-ils généralement moins élevés sur Internet?

- Les cyberconsommateurs sont-ils plus sensibles à de timides modifications du prix sur Internet?

- Les commerçants ajustent-ils leur prix plus souvent et plus sensiblement sur Internet?

- Les écarts entre les prix les moins élevés et les plus élevés sont-ils moins importants sur Internet?

Bien que ces auteurs n'aient étudié que les prix d'articles tels que les livres et les disques, les résultats montrent en effet que les prix de ces produits sont généralement moins élevés sur Internet ${ }^{54}$, que les cyberconsommateurs sont plus sensibles à de timides modifications du prix sur Internet, que les écarts entre les prix les moins élevés et les plus élevés sont moins importants sur Internet et finalement, que les commerçants proposent certes des prix légèrement inférieurs sur Internet par rapport aux boutiques traditionnelles, mais que cette stratégie ne rime pas avec une augmentation des ventes pour autant. Voici donc une réponse intéressante pour les commerçants désireux d'ouvrir une boutique virtuelle et ne sachant pas quelle stratégie adopter en matière de positionnement au niveau du prix.

Cependant, quel est le réel intérêt d'un consommateur qui, même s'il gagne quelques dollars en achetant par Internet, ne peut jouir de son produit immédiatement, si ce n'est de l'effet de mode duquel il pourra se vanter? Au cours de l'année 2001, 13 \% des Québécois ont en effet acheté en ligne, ce qui représente une légère hausse par rapport à l'année précédente ${ }^{55}$.

Par ailleurs, pour les fournisseurs d'information gratuite à destination des consommateurs ou d'autres entreprises, ils peuvent être tentés par les modèles hybrides, consistant à divulguer gratuitement de l'information sur un portail ou un site traditionnel, tout en s'assurant des revenus substantiels grâce aux redevances des annonceurs.

Une des principales difficultés, en ce qui a trait à cette variable, est de proposer des modèles encore trop avantgardistes, si bien que les consommateurs, tout comme les annonceurs ou les commerçants, ne croient pas à sa viabilité. Espérons toutefois - et nous croyons que cela est possible en raison du jeune âge de l'Internet - que de 
nombreuses autres innovations feront leur apparition en proposant des modèles plus évolués encore.

\section{L'utilisation d'un modèle d'affaires élec- tronique sur Internet ne doit pas déroger des règles traditionnelles de fixation des prix : les mêmes principes s'appliquent, que l'on soit dans le monde physique ou virtuel.}

Avant de conclure sur cette variable, il nous semble intéressant de préciser au lecteur que l'utilisation d'un modèle d'affaires électronique sur Internet ne doit pas déroger des règles traditionnelles de fixation des prix : les mêmes principes s'appliquent, que l'on soit dans le monde physique ou virtuel. À ce chapitre, d'ailleurs, Bégin et autres ${ }^{56}$ rapportent le commentaire d'un dirigeant d'entreprise ayant un site $\mathrm{Web}^{57}$ et qui précisait à juste titre: «Quand on fait du e-commerce, c'est le commerce qui est important, pas le $e »$.

\section{Notes et références}

1 William Menvielle est étudiant au doctorat en administration des affaires à l'Université du Québec à Trois-Rivières, Denis Pettigrew et Jocelyn D. Perreault sont professeurs de marketing, également à l'Université du Québec à Trois-Rivières.

2 TIMERS, P. (1998). «Business Models for Electronic Markets», Electronic Markets, vol. 8, $\mathrm{n}^{\circ} 2$.

3 Business to Business

4 Business to Consumer

5 TIMERS, P. (1998). Op. cit., note 2.

6 PEPPERS, D., M. ROGERS and B. DORF (1999). «Is your company ready for one-to-one marketing?», Harvard Business Review, Boston, janvier-février.

7

http://www.merx.ca

8 http://encan.canoe.com

9 http://www.123vendu.com

$10 \mathrm{http} / / / \mathrm{www}$. fastparts.com

11 Littéralement «brick and mortar» dans la littérature anglophone.

12 http://www.mallcanada.com

13 http://www.fortune1000.ca/cce/centre.htm

14 TIMERS, P. (1998). Op. cit., note 2.

15 http://www.altavista.com

16 http://www.copernic.com

17 http://www.cybion.fr
18 BRUDEY et DUCROQ (1998). Cités dans VOLLE, P. (1999). «Du marketing des points de vente à celui des sites marchands : spécificités, opportunités et questions de recherche», Cahier de recherche $n^{\circ}$ 276, Centre de recherche DMSP, Université Dauphine, Paris, juillet.

19 http://www.uqtr.uquebec.ca/inrpme

20 http://www.comm-qc.gouv.qc.ca/default.asp

21 Par consommateur, on entend ici à la fois le particulier et l'entreprise.

22 http://www.nua.ie/surveys

23 http://www.branchez-vous.com

24 http://www.monentreprise.com http://www.radio-Canada.ca/nouvelles

25 http://www.archambault.ca

26 www.pooddles.com

27 http://www.candanantire.ca

28 SCHWARTZ, E. I. (1996). «Advertising Webonomics 101», Electrosphere, Wired, $\mathrm{n}^{\circ} 4.02$, [www.wired.com/wired4.02/webonomics.html], février.

29 Terme québécois désignant le «chat».

30 DUFOUR, A. (1998). Du marketing au cybermarketing: proposition d'un cadre méthodologique pour intégrer Internet dans la stratégie de l'entreprise, Thèse de doctorat,École des Hautes Études Commerciales, Lausanne.

31 http://www.microsoft.com

32 BAKOS, Y. (1998). «The emerging role of electronic marketplaces on the Internet», Communications of the ACM, août.

33 http://www.lactualite.com

34 REYNOLDS, J. (2000). « The consumer marketspace», International Journal of Retail \& Distribution Management, vol. 28, $\mathrm{n}^{\circ} 10$.

35 BAKOS, Y. (2001). « The emerging lanscape for retail E-commerce», Journal of Economic Perspectives, janvier.

36 http://www.sitesell.com/

37 http://www.geis.com/index.jsp

38 Request for Proposal: demande de propositions de prix (traduction libre).

39 Consumer to Business : Consommateur à Entreprise.

40 http://www.priceline.com

41 http://www.freemarkets.com

42 http://www.esterel.com/

43 BAKOS, Y. (1998). Op. cit., note 32.

44 RAYMOND, L. et W. MENVIELLE (2000). Gestion des technologies de l'information et commerce électronique dans la 
$P M E$, Rapport de veille-synthèse présenté à Développement économique Canada.

45 Par ailleurs, le consommateur peut rechercher le meilleur prix pour un produit en allant sur divers sites ou confier cette tâche à des agents intelligents, de petits moteurs de recherche paramétrables qui réaliseront cette tâche pour lui (ce sont les célèbres Shoping Robot, plus connus sous le néologisme Shopbot).

46 http://www.jango.com

47 http://ecommerce.media.mit.edu/kasbah

48 http://ecommerce.media.mit.edu/tete-a-tete

49 http://www.copernic.com/fr/products/shopper/index.html

50 http :auction.eecs.umich.edu

51 HANSON (2000). Cité dans JALLAT, F. (2001). «Sociologie du prix sur Internet», Décisions Marketing, n 22 , janvier-avril.

52 Alors que les variables «prix », «promotion» et «place» engendrent des coûts, la variable «prix» est celle permettant à l'entreprise de venir les couvrir et de dégager un bénéfice.
53 SMITH, M. D., J. BAILEY and E. BRYNJOLFSSON (1999). «Understanding Digital Markets», in E. Brynjolfsson and B. Kahin, Understanding the Digital Economy, MIT Press,Boston.

54 Pour pouvoir comparer équitablement les prix sur un marché traditionnel et sur Internet, il conviendrait d'inclure, pour le marché traditionnel, les coûts liés à l'acte d'achat: coût d'utilisation du moyen de transport, coût du temps passé à magasiner, etc., mais peu de consommateurs sont aussi rationnels. Toutefois, il convient de bien comparer les prix des produits lorsque le consommateur vise un achat sur l'un ou l'autre des moyens utilisés : l'achat d'un ordinateur sur le site Web de Dell engendre des coûts de manutention de plus de 150 \$! C'est le prix d'une bonne imprimante pour une configuration achetée dans une boutique informatique.

55 LACROIX, E. (2002). NETendances 2001 : utilisation d'Internet au Québec, CEFRIO, Montréal.

56 BEGIN et autres (2001). «Études de cas sur les pratiques de commerce électronique des entreprises québécoises, Cahier de recherche CICMA 01-06, École des Hautes Études Commerciales, Montréal.

57 www.VolvoWorld.com 